\title{
Wireless Sensor Network Based Epileptic Seizure Detector $^{+}$
}

\author{
Mohammad Monirujjaman Khan *, Tahia Tazin, Fazle Rabbi Mithun, Tasnova Tabassum and \\ Md Adnan Chowdhury \\ Department of Electrical and Computer Engineering, North South University, Bashundhara, Dhaka 1229, \\ Bangladesh \\ * Correspondence: monirujjman.khan@northsouth.edu; Tel.: +880-1779006296 \\ + Presented at the 7th International Electronic Conference on Sensors and Applications, 15-30 November \\ 2020; Available online: https://ecsa-7.sciforum.net/.
}

Published: 15 November 2020

\begin{abstract}
Epilepsy is a focal sensory (neurological) disease in which cerebrum movement gets abnormal, spark off seizures or period of surprising conduct, sensations, and occasionally the loss of consciousness. The diagnosis of epileptic seizures is chiefly done by methods for electroencephalogram (EEG) observing. Even though this technique is precise, it isn't easy for the patient because the EEG-anodes must be appended to the scalp which is uncomfortable for the patient. Seizure indications can fluctuate broadly. A few people with epilepsy just gaze vacantly for a couple of moments during a seizure, while others over and again jerk their arms or legs. This makes constant monitoring at home significantly more troublesome. Epilepsy patients are often unable to move freely for fear of convulsions. After analyzing all the issues related to epilepsy, we have proposed a plan to make a cheap and usable device as a solution so that an epileptic patient can move like a normal human being. With this device, if an epileptic patient falls ill anywhere, his or her information will be sent to the nearest hospital and his emergency contact numbers in the form of SMS.
\end{abstract}

Keywords: epilepsy; seizure; EEG; hospital; emergency; patients

\section{Introduction}

Epilepsy is an immediate non-communicable malady of the cerebrum that can infect all aged people. The majority of the affected individuals can be dealt with effectively with drug treatment $(67 \%)$ or neurosurgical strategies (7-8\%). All things considered, $25 \%$ of the affected individuals can't be treated by any accessible treatment [1]. For unmanageable patients who keep on having successive seizures, it has been indicated that escalated checking with an electroencephalogram (EEG) and video over a significant stretch, adds to the administration of everyday care and the change of medication treatment [2]. The drawn-out observing with EEG and video can be exceptionally horrendous for patients, and investigating a lot of EEG/video-information is very work seriously for clinical faculty. Moreover, this technique cannot yet to be applied continuous methodology.

All the previously mentioned factors have made it important to search for sensors that show restraint neighborly and can be utilized for a solid programmed identification of epileptic seizures. One of these sensors is the accelerometer. Accelerometers are utilized in numerous clinical exploration territories for action acknowledgment [3-5]. For this reason, we will create a device using a pulse sensor, Arduino, GSM, and other Wi-Fi modules with an accelerometer which will make it easier to detect seizures and send information to the nearest hospital. Our device will be more usable at a lower price. And using this device in the future will also be able to reduce accidents caused by epilepsy. 


\section{Materials and Methods}

A hand-band that recognizes seizures, will be outfitted with a pulse meter, gyroscope sensor, and also will interface with $\mathrm{Wi}-\mathrm{Fi}$ to report the overall imperatives and attributes of the framework which will be recorded as follows:

- All outer wirings will be taken out to permit the subject under test move without limitations.

- The organization convention will permit the sensor organization to introduce itself in an exceptionally specially appointed, self-arranging way.

- While information unwavering quality will be consistently significant, it will turn into a basic prerequisite for some applications, for instance, in clinical checking.

In Figure 1, the flowchart of the proposed system has been shown.

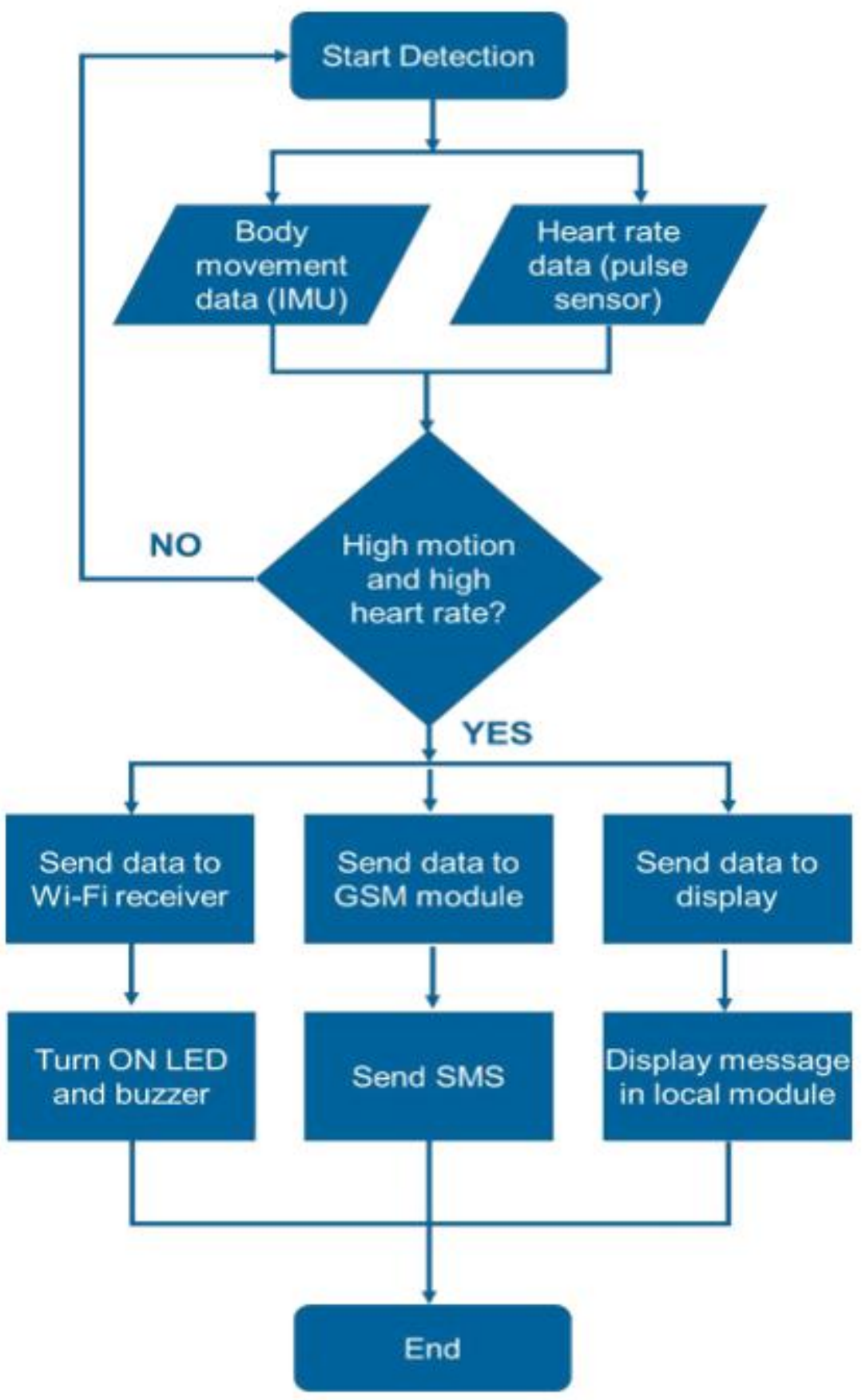

Figure 1. Flowchart of the proposed system. 
In this framework, there will be 2 sections. The first one is the detection part and the second one is the notification part. Firstly, we will take the heart rate of the patients as input. When seizer occurs, the patient's body will vibrate and the whole body will fall. Using the triple-axis accelerometer, the system will detect the vibration and the fall down of the patient. But also, at that same time, the heart rate of patient will be jumping up and down which is known as irregular arrhythmia. So, by using a heart rate sensor the system will be able to detect this event. From both of those sensors, the system will send the analog signal as a single input in the microprocessor. Then it will set some conditions so that the detection can be more accurate. Datasets from patients experiencing substantial epilepsy will be utilized for the advancement of a programmed identification calculation. Patients will be solicited to play out a grouping from ordinary typical exercises however they won't be able to know about the trial. Ordinary exercises that we will be recorded included static exercises, for example, perusing, working with a PC, brushing of teeth, and eating, and dynamic exercises, for example, strolling. Interestingly with body advancements, the commotion from the sensor and assessment system will be disregarded. All data will be used in this assessment which will be recorded while the patients will be in their living atmosphere, along these lines there will no speeding up conveyed by external sources. When there will be no turn of events, physiological disturbs, like inhale and beat and gravitational speeding up will be observable in the sign.

In Figure 2, the basic diagram of the detection side has been shown. Two sensors will take the analog signal from the patient and process it into a single input in the microprocessor. If both sensors sense high and abnormal value at the same time, then the microprocessor will give an output that seizure is detected through display. Two types of wireless communication technologies will be used such as GSM and Wi-Fi. Using GSM, SMS will be sent to a pre-programmed number. Wi-Fi sends signal to another Wi-Fi on the notification side.

On the other hand, by using wireless communication, this device will send SMS, display the information and alarm the emergency persons. The output of the detection part will be the input of the notification part. If the detection part detects any seizure then using Wi-Fi and GSM module, it will send the data to a phone and another microprocessor. In that microprocessor output, a display and alarm will be connected. The basic diagram of the notification part has been given in Figure 3 . The input of the notification part will be the output of the detection side of the Wi-Fi.

When the detection side Wi-Fi will send any signal, then the receiver side Wi-Fi will receive the signal and acts as an input of the microcontroller. The pre-program microcontroller will show the detection information through the display which will be connected to the microcontroller. The is also a buzzer that will be turned on if a seizure is detected.

Therefore, the whole system will be operated in one logic, if the patient body vibrates and his or her heart rate act abnormally then the detection side will send a signal to the notification side, if not the notification side will not show any information. 


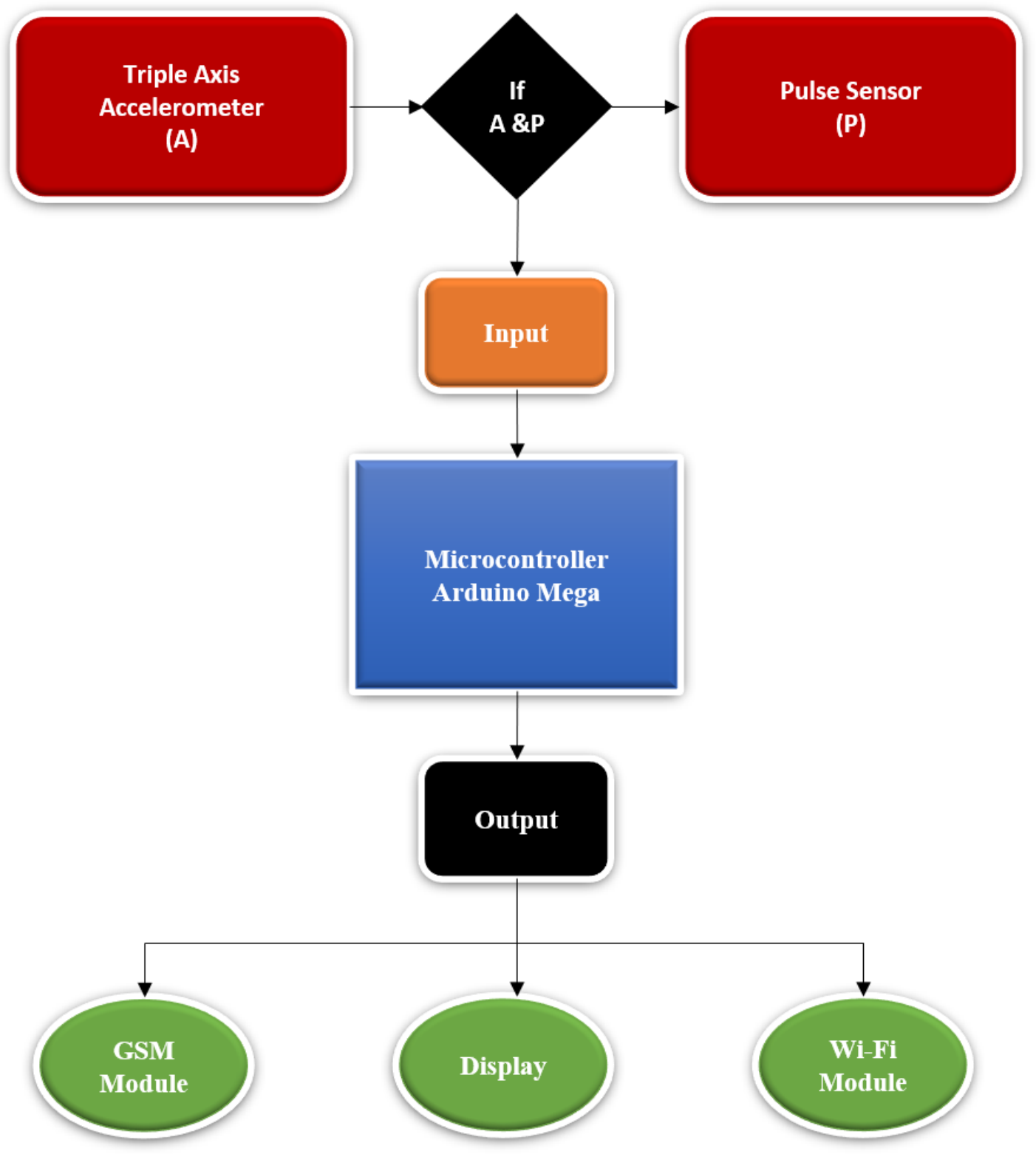

Figure 2. Detection part of the system. 


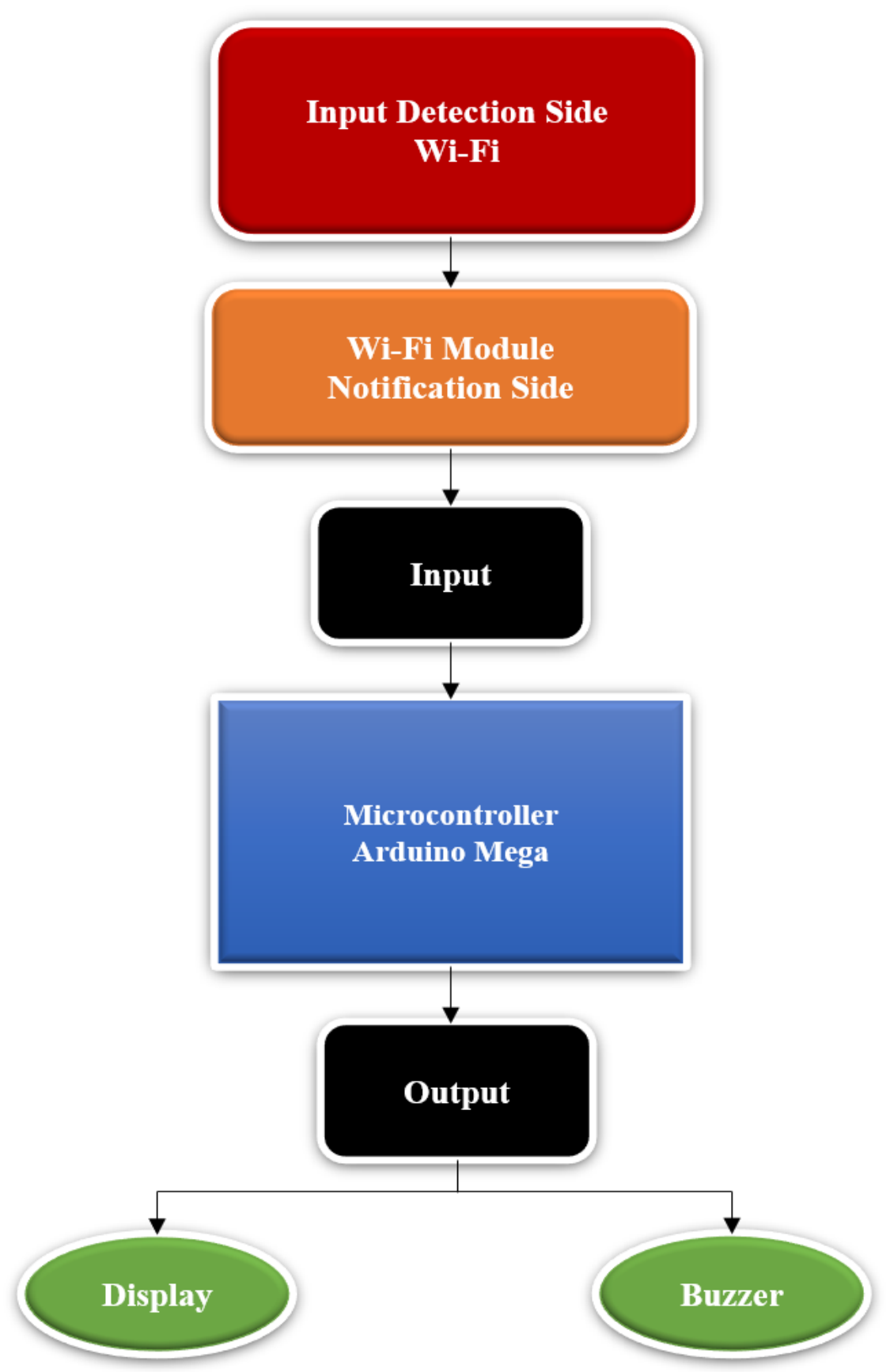

Figure 3. Notification part of the system.

\section{Hardware}

We will use the hardware mentioned in Table 1 to make this device.

Table 1. Required hardware components.

\begin{tabular}{cc}
\hline Name of the Parts & Quantity \\
\hline Arduino Mega & 2 \\
Triple Axis Accelerometer & 1 \\
Pulse Sensor & 1 \\
ESP8266 Wi-Fi Module & 2 \\
GSM Module & 1 \\
Display $(16 \times 2)$ & 2 \\
Wire & - \\
\hline
\end{tabular}




\section{Future Plan and Conclusions}

The most fascinating use of WSN is for health checking. In this paper, we propose an epilepsy checking framework dependent on WSN for the discovery of epilepsy seizures. This framework will be utilized for patients living in a clinical climate or at their home, where they do their day by day schedule. This framework will decide the area of the patient when a seizure will be distinguished and sends an alert to the emergency clinic staff or the patient's family members. Since the sensors are remote, the subject under test can move without limitations. Moreover, because of the little size of the sensor hubs, they will be wearable for patients. A lightweight, easy, savvy wearable gadget will be created so that it can help a huge number of casualties of epilepsy around the world. With this proposed system, epilepsy patients can be lived independently like ordinary individuals without any stress. Also, it will be a very cheap and user-friendly system so it will eventually help poor people all over the world. Most importantly, this system will make the epilepsy patient independent.

We have started work on making this device. In the future, we will publish the results in an MDPI journal. Epilepsy causes many accidents in our country every day. Although many researchers have studied epilepsy, they have not been able to predict its onset in any way. We will create a framework using deep learning in our future work so that epilepsy patients can be aware in advance.

Funding: This research received no external funding.

Acknowledgments: Authors would like to thank Department of Electrical and Computer Engineering of North South University.

Conflicts of Interest: The authors declare no conflict of interest.

\section{References}

1. Witte, H.; Iasemidis, L.D.; Litt, B. Special issue on epileptic seizure prediction. IEEE Trans. Biomed. Eng. 2005, 50, 537-539.

2. Binnie, C.D.; Aarts, J.H.; Van Bentum-De Boer, P.T.; Wisman, T. Monitoring at the institute for epilepsy fight in Bosch. Electroencephalogr. Clin. Neurophysiol. Suppl. 1985, 37, 341-355.

3. Mathie, M.J.; Celler, B.G.; Lovell, N.H.; Coster, A.C. Classification of basic daily movements using a triaxial accelerometer. Med. Biol. EngComput. 2004, 42, 679-687.

4. Veltink, P.; Bussmann, H.B.; de Vries, W.; Martens, W.L.; Van Lummel, R.C. Detection of static and dynamic activities using uniaxial accelerometers. IEEE Trans. Rehabil. Eng. 1996, 4, 375-385.

5. Najafi, B.; Aminian, K.; Paraschiv-Ionescu, A.; Loew, F.; Bula, C.; Robert, P. Ambulatory system for human motion analysis using a kinematic sensor: Monitoring of daily physical activity in the elderly. IEEE Trans. Biomed. Eng. 2003, 50, 711-723.

Publisher's Note: MDPI stays neutral with regard to jurisdictional claims in published maps and institutional affiliations.

(C) 2020 by the authors. Submitted for possible open access publication under the terms and conditions of the Creative Commons Attribution (CC BY) license (http://creativecommons.org/licenses/by/4.0/). 